1 in 24 children were reported to have delayed presentation during lockdown and a small number of these children required PICU admission.

Overall the data are reassuring that the majority of children are brought to ED appropriately. Ongoing messaging for parents regarding red and amber symptoms continues to be important, particularly in the event of any further lockdowns.

\section{SIGNIFICANT IMPACT OF SARS-COV-2 ON ATTENDANCES, BUT NOT ACUITY, IN A CHILDREN'S EMERGENCY DEPARTMENT}

${ }^{1}$ Damian Roland, ${ }^{2}$ William Jones. ${ }^{1}$ Leicester University and Hospitals; ${ }^{2}$ University Hospitals of Leicester NHS Trust

\subsection{6/emj-2020-rcemabstracts.52}

Aims/Objectives/Background There have been anecdotal reports that delay in presenting to Children's Emergency Departments (CED) during COVID19, combined with a reduction in more minor cases, has increased the relative acuity of children in CED.

Methods/Design In order to determine the impact of SARS$\mathrm{CoV}-2$ on our tertiary CED we compared attendances, diagnosis at discharge, initial paediatric observation priority score (POPS) and Healthcare Resource Group (HRG) code for the months of March, April and May 2018, 2019 and 2020. POPS is a bespoke early warning-like score and is calculated between $0-16$ ( 0 low acuity;16 high acuity) based on 8 components including vital signs and subjective observations. A large proportion of children presenting have an unrecorded or POPS 0 as they are an injury so a POPS of greater than 0 was used in analysis. A lower HRG scores indicates increased resource utilisation.

Results/Conclusions Presentations in March, April and May 2020 fell compared with previous years with overall HRG weighting slightly increasing to 8.71 from 8.48 (2019) and 8.98 (2018) which is a clinically negligible change. There was a trend for a greater proportion of children with a POPS 0 in 2020 compared to previous years. Mean and median POPS was unchanged (table 1). Children diagnosed with an infectious disease fell by 30.5 attendances per 1000 presentations and soft tissue injuries fell by 10.0 attendances per 1000 presentations comparing 2020 and 2019. There were increases in codes relevant to specific paediatric diagnoses (croup, febrile convulsion etc.) by 11.4 attendances per 1000 presentations in the same time period and fracture/dislocations increased by 9.1 attendances per 1000 presentations.

While having a huge impact on childrens' presentations to Emergency Departments there is no evidence, in our institution, of significantly elevated acuity, or a large change in casemix, as a result of the COVID19 pandemic compared to previous years.

\section{2 'TAP IT': TRAINEE LED INITIATIVE TO REDUCE UNNECESSARY BLOOD TESTING IN A PAEDIATRIC EMERGENCY DEPARTMENT}

${ }^{1}$ Eliza Magnusen, ${ }^{2}$ Kene Maduemem, ${ }^{2}$ Ayesha Shafiq, ${ }^{3}$ Daniel Crankshaw, ${ }^{1}$ Habab Mekki, ${ }^{1}$ Arani Sridhar. 'Leicester Royal Infirmary; ${ }^{2}$ Northampton General Hospital; ${ }^{3}$ John Radcliffe Hospital Oxford

\subsection{6/emj-2020-rcemabstracts. 53}

Aims/Objectives/Background Unnecessary blood testing in the paediatric emergency department (PED) is a potential starting point for diagnostic dilemma, anxiety to families and increased healthcare costs. We hypothesized that a significant number of blood tests are performed instinctively rather than clinically indicated. This stimulated a quality improvement initiative to enlighten trainees on the utility of blood tests while aiming to enhance clinical decision making.

Methods/Design Children presenting to the acute care team in a tertiary PED who had blood tests over a 2 -week period in April 2019 were enrolled. Blood tests requested were interpreted in line with presenting features and clinical impression. Following implementation of changes (posters, QI champions, educational sessions), a repeat analysis was done over a 2 week period in October 2019.

Results/Conclusions One hundred and one children in the first cycle were enrolled. Blood testing analysis revealed that $70 \%$, 47\%, and 32\% had liver bloods, bone profile, and clotting testing done, respectively. Over half of these blood tests had no clear clinical indication. The yield of the tests performed without clinical indication was $0 \%$. Case vignettes were attempted by trainees and ANPs to evaluate their attitudes to blood test requests. Number of requested blood tests not clinically indicated was lower than anticipated; probably explained by self-thought processes. Blood tests performed on 100 children in the second cycle demonstrated a significant reduction in tests done without clear clinical

\begin{tabular}{|c|c|c|c|c|c|c|c|c|}
\hline Month & $\begin{array}{l}\text { Year of Arrival } \\
\text { Date }\end{array}$ & $\begin{array}{l}\text { Attendance } \\
\text { Count }\end{array}$ & $\begin{array}{l}\text { Number of Children with a POPS }>0 \\
(\%)\end{array}$ & $\begin{array}{l}\text { Attendance } \\
\text { Count }\end{array}$ & $\begin{array}{l}\text { Mean } \\
\text { EWS }\end{array}$ & $\begin{array}{l}\text { Median } \\
\text { EWS }\end{array}$ & $\begin{array}{l}\text { 25th } \\
\text { Percentile }\end{array}$ & $\begin{array}{l}\text { 75th } \\
\text { Percentile }\end{array}$ \\
\hline March & 2018 & 4,123 & $1,682(40.8)$ & 4,123 & 5 & 2.00 & 1 & 3 \\
\hline March & 2019 & 5,738 & $2,443(42.3)$ & 5,738 & 6 & 2.00 & 1 & 3 \\
\hline March & 2020 & 3,831 & $1,638(42.8)$ & 3,831 & 6 & 2.00 & 1 & 3 \\
\hline April & 2018 & 4,134 & $1,436(34.7)$ & 4,134 & 5 & 2.00 & 1 & 3 \\
\hline April & 2019 & 5,159 & $2,066(40.1)$ & 5,159 & 5.5 & 2.00 & 1 & 3 \\
\hline April & 2020 & 1,625 & 778 (47.9) & 1,625 & 4.75 & 1.00 & 1 & 2 \\
\hline May & 2018 & 4,594 & $1,515(33.0)$ & 4,594 & 6.09 & 2.00 & 1 & 3 \\
\hline May & 2019 & 4,957 & $1,887(38.1)$ & 4,957 & 6.09 & 2.00 & 1 & 3 \\
\hline May & 2020 & 2,312 & $937(40.5)$ & 2,312 & 5.8 & 1.00 & 1 & 2 \\
\hline
\end{tabular}


indication. Liver bloods, clotting screen, bone profile tests were performed on $40 \%, 24 \%$ and $27 \%$ respectively in the second cycle.

The utility of blood tests may be an under recognised subject in paediatric training which needs addressing. This project typified positive impact of culture change via QI champions and educational sessions. Implementation of such changes is sustainable with an estimated savings of at least $£ 500 /$ month.

\section{CHILDREN'S EMERGENCY DEPARTMENT REVIEW CLINIC: WHAT WE HAVE LEARNT IN THE COVID-19 PANDEMIC}

Charlotte Munday, Neha Jain, Seamus Harrington, Benjamin Darvill, Sophie Perry, Alice Irwin, Francesca Deibel, Richard Turck, Clarissa Barber. Bristol Royal Hospital for Children

\subsection{6/emj-2020-rcemabstracts.54}

Aims/Objectives/Background The Children's Emergency Department (CED) in the Bristol Royal Hospital for Children (BRHC) runs 'Review Clinics' by a Paediatric Emergency Consultant. These were suspended in March 2020 due to the Covid-19 pandemic and new patient pathways were put in place. Our aim was to assess the effectiveness of these pathways and to evaluate if they should continue once the pressures of Covid-19 have eased.

Methods/Design We reviewed all the attendances to the ED Review Clinic over a six-week period in 2019 and identified common conditions that are seen in this clinic - abscesses, cellulitis, scaphoid fracture, toddler fracture, limp, headache, peri-orbital cellulitis, torticollis, and neck lumps (accounting for $66 \%$ of all attendances).

We then identified all of the ED attendances with these conditions over the same six-week period during the Covid-19 pandemic and performed a service review using medical notes. Results/Conclusions All children presenting with a limp during the Covid-19 pandemic were discharged with a leaflet and advised to return in one week if the limp persisted. None of them re-attended.

Scaphoid and toddler fractures that presented during the Covid-19 pandemic were followed-up by Orthopaedics with a combination of telephone and face-to-face reviews. The toddler fractures were put into soft casts to be removed at home - 93\% of parents were happy to do this. Orthopaedic review was quicker than the previous ED Review Clinic (an average of 63 hours compared to 216 for scaphoid fractures and 67 hours compared to 235 for toddler fractures).

The results suggest that ED Review Clinics can and should change. Children with a limp can be sent home safely with clear advice. There is a greater role for telephone reviews, in particular, for injuries such as toddler fractures. This would be quicker, less time-consuming for patients, parents and physicians and more cost effective for the department.

\section{PRESENTATION OF VULNERABLE CHILDREN TO ED DURING LOCKDOWN: A CONCERNING CONSEQUENCE OF ISOLATION}

Lalarukh Asim, Raeesa Jina, Sreena Das. King's College Hospital
Aims/Objectives/Background This study aims to evaluate changes to child safeguarding attendances to the emergency department (ED) during the lockdown period 1st of March till 31st May 2020 compared to the same time period in 2019.

The COVID-19 pandemic is the defining health crisis of our time and the first time firm social restrictions have been imposed since safeguarding practices have become embedded in the NHS. The NSPCC reported a large increase in contacts but there remain concerns that vulnerable children are invisible to agencies during this time.

Methods/Design All children ( $<18$ years) who attended King's College Hospital's ED, and were reviewed in the weekly ED safeguarding meeting were included. Data was collected from electronic patient records for different parameters.

Results/Conclusions The total number of children presenting via ED for safeguarding review fell from 865 in 2019 to 355 for the same period in 2020. However, the proportion requiring action by the hospital safeguarding team showed a significant increase $\left(p=1.5 \times 10^{-4}\right)$ suggesting the severity of cases during COVID-19 is worse.

The percentage of stabbings doubled $(\mathrm{p}=0.04)$ despite lockdown measures. This may be a contributor to the significant increase in referral to youth workers $\left(p=8 \times 10^{-4}\right)$. The number of children attending who were considered high risk due to previous safeguarding concerns dropped by $75 \%$.

As expected, the proportion of household injuries such as accidental ingestion and burns showed a significant increase $(p=0.01$ and $p=0.002$ respectively). The proportion of children from outside of our local boroughs was surprisingly higher in $2020(p=0.03)$.

The findings show that the number of cases triggering a safeguarding review has dropped during lockdown and raises concerns about vulnerable children who remain hidden. The findings also suggest an increased severity of safeguarding presentations, supporting our fears that the implications of lockdown on vulnerable children is yet to be realised.

\section{THE DIAGNOSTIC ROLE OF SHUNT SERIES RADIOGRAPHS (SSR) IN CHILDREN PRESENTING TO THE CHILDREN'S EMERGENCY DEPARTMENT (CED) WITH SUSPECTED VENTRICULOPERITONEAL (VP) SHUNT FAILURE}

${ }^{1}$ Angharad Griffiths, ${ }^{1}$ Ikechukwu Okafor, ${ }^{2}$ Thomas Beattie. ${ }^{1}$ Children's Health Ireland at Temple Street; ${ }^{2}$ University of Edinburgh

10.1136/emj-2020-rcemabstracts.56

Aims/Objectives/Background VP shunts are used to drain CSF from the cranial vault because of a wide range of pathologies and, like any piece of hardware, can fail. Traditionally investigations include SSR and CT. This project examines the role of SSR in evaluating children with suspected VP shunt failure.

Primary outcome: Sensitivity and specificity of SSR in children presenting to the CED with concern for shunt failure. Methods/Design Conducted in a single centre, tertiary CED of the national Irish Neurosurgical(NS) referral centre (ED attendance: $>50,000$ patients/year). 100 sequential SSR requested by the $\mathrm{CED}$ were reviewed. Clinical information was extracted from electronic requests. Shunt failure was defined by the need for NS intervention(Revision). 